\title{
MULTIVARIATE ORTHOGONAL POLYNOMIALS AND OPERATOR THEORY
}

\author{
YUAN XU
}

\begin{abstract}
The multivariate orthogonal polynomials are related to a family of commuting selfadjoint operators. The spectral theorem for these operators is used to prove that a polynomial sequence satisfying a vector-matrix form of the three-term relation is orthonormal with a determinate measure.
\end{abstract}

\section{INTRODUCTION}

The purpose of this paper is to use the operator theory to study the multivariate orthogonal polynomials. First let us recall the relevant one variable theory of orthogonal polynomials (cf. $[5,16])$. tions

Let $\left\{p_{n}(x)\right\}_{n=0}^{\infty}$ be a sequence of polynomials satisfying the three-term rela-

$$
x p_{n}(x)=a_{n} p_{n+1}(x)+b_{n} p_{n}(x)+a_{n-1} p_{n-1}(x) .
$$

Then Favard's Theorem states that the sequence $p_{n}$ is orthonormal with respect to a measure $\mu$ if and only if $a_{n}$ are positive real numbers. It is known that we can relate the measure of orthogonality to the spectral measure of a selfadjoint operator; the matrix representation of this operator is a Jacobi matrix (cf. [6, 15]). Therefore it is possible to use operator theory to study the nature of the measure (cf. $[6,7])$. In particular, one can prove that the measure is of compact support if and only if $\left\{a_{n}\right\}$ and $\left\{b_{n}\right\}$ are bounded. However, the usual way of proving the latter result (cf. [5]) does not use operator theory, but a more elementary method which requires the knowledge of the zeros of orthogonal polynomials.

The theory of multivariate orthogonal polynomials is far from complete. There are only a few papers in the literature dealing with the general multivariable theory (cf. $[4,9,10,17])$. In a recent paper [17], we have proved an extension of Favard's Theorem, where the orthogonality is with respect to a quasi-inner product (see §2). Earlier results can be found in [9]. The three-terms relation now takes the vector-matrix form

$$
x_{i} \mathbb{P}_{n}(\mathbf{x})=A_{n, i} \mathbb{P}_{n+1}(\mathbf{x})+B_{n, i} \mathbb{P}_{n}(\mathbf{x})+A_{n-1, i}^{T} \mathbb{P}_{n-1}(\mathbf{x}), \quad 1 \leq i \leq d,
$$

where $\mathbf{x}=\left(x_{1}, \ldots, x_{d}\right)^{T} \in \mathbf{R}^{d}, \mathbb{P}_{n}$ is a polynomial vector, and $A_{n, i}$ and $B_{n, i}$ are matrices (see §2). One problem for the multivariate orthogonal polynomials

Received by the editors May 14, 1992.

1991 Mathematics Subject Classification. Primary 42C05, 47B15.

Key words and phrases. Multivariate orthogonal polynomials, recurrence relation, commuting selfadjoint operators, determinate measure. 
is that the zeros are actually common zeros of a family of polynomials, thus, the elegant theorems for zeros in the one variable case are no longer available. However, as we shall show, the multivariate orthogonal polynomials are related to a family of commuting selfadjoint operators. The purpose of this paper is to establish this relation. In particular, we shall prove that if the norms of matrices in the three-terms relation are bounded, then the set of polynomials is orthonormal with respect to a nonnegative measure with compact support. In a subsequent paper the nature of the measure will be studied via the operator approach.

The paper is organized as follows. In $\S 2$ we introduce the notation and present the basic results of multivariate orthogonal polynomials. The main results are stated in $\S 3$. The operator approach and the proof of the main theorem are in $\S 4$.

\section{MultivaRIATE ORTHOGONAL POLYNOMIALS}

Let $\mathbf{N}_{0}$ be the set of nonnegative integers. For $\alpha=\left(\alpha_{1}, \ldots, \alpha_{d}\right) \in \mathbf{N}_{0}^{d}$ and $\mathbf{x}=\left(x_{1}, \ldots, x_{d}\right) \in \mathbf{R}^{d}$ we write $\mathbf{x}^{\alpha}=x_{1}^{\alpha_{1}} \cdots x_{d}^{\alpha_{d}}$. The number $|\alpha|=$ $\alpha_{1}+\cdots+\alpha_{d} \in \mathbf{N}_{0}$ is called the total degree of $\mathbf{x}^{\alpha}$. For $n \in \mathbf{N}_{0}$ we denote by $\Pi_{n}^{d}$ the set of polynomials of total degree at most $n$ in $d$ variables, and $\Pi^{d}$ the set of all polynomials in $d$ variables.

A real valued linear functional $\mathscr{L}$ is said to induce a quasi-inner product on $\Pi^{d}$ by the formula $\langle P, Q\rangle=\mathscr{L}(P Q)$ provided there exists a basis $B$ of $\Pi^{d}$ such that

$$
\mathscr{L}(P Q) \begin{cases}=0, & \text { if } P \neq Q, \\ \neq 0, & \text { if } P=Q, \forall P, Q \in B,\end{cases}
$$

and $B=\left\{P_{j}^{k}\right\}_{k=0}^{\infty} r_{j=1}^{d}$, where the superscript $k$ means that $P_{j}^{k}$ is of total degree $k$ and $r_{k}^{d}=\operatorname{dim} \Pi_{k}^{d}-\operatorname{dim} \Pi_{k-1}^{d}$. We take $\mathscr{L}\left(P^{2}\right)=1$ in $(2.1)$ when $\mathscr{L}$ induces an inner product. Two polynomials $P$ and $Q$ are said to be orthogonal to each other with respect to $\mathscr{L}$ if $\mathscr{L}(P Q)=0$. For each $k \in \mathbf{N}_{0}$, let $V_{k}^{d} \subset \Pi_{k}^{d}$ denote the set of polynomials of degree exactly equal to $k$, together with zero, that are orthogonal to all polynomials in $\Pi_{k-1}^{d}$. Then $V_{k}$ is a vector space of dimension $r_{k}^{d}=\left(\begin{array}{c}k+d-1 \\ k\end{array}\right)$, and $V_{k}$ 's are mutually orthogonal. Throughout this paper, the letter $d$ is reserved for the number of variables or the dimension. It is fixed and will be omitted sometimes. For example, we write $r_{k}$ for $r_{k}^{d}$ in the following. For a sequence of polynomials $\left\{P_{j}^{k}\right\}_{j=1}^{r_{k}}$ of total degree $k$, we introduce the vector notation

$$
\mathbb{P}_{k}(\mathbf{x})=\left[P_{1}^{k}(\mathbf{x}), P_{2}^{k}(\mathbf{x}), \ldots, P_{r_{k}}^{k}(\mathbf{x})\right]^{T} .
$$

If a matrix $P=\left(p_{i j}\right)$ is given whose elements $p_{i j}$ are polynomials in $\Pi^{d}$, we denote by $\mathscr{L}(P)$ the matrix whose elements are the values $\mathscr{L}\left(p_{i j}\right)$. For our convenience, if $\left\{P_{j}^{k}\right\}_{j=1}^{r_{k}}$ is a basis for $V_{k}^{d}$, we shall say that $\mathbb{P}_{k}$ is a basis for $V_{k}^{d}$ and that $\left\{\mathbb{P}_{k}\right\}_{k=0}^{\infty}$ is an orthogonal basis for $\Pi^{d}$. If $\mathscr{L}$ is an inner product, $\left\{\mathbb{P}_{k}\right\}_{k=0}^{\infty}$ is orthogonal with respect to $\mathscr{L}$ and $\mathscr{L}\left(\mathbb{P}_{k} \mathbb{P}_{k}^{T}\right)=I_{r_{k}}$, then we say $\left\{\mathbf{P}_{n}\right\}_{n=0}^{\infty}$ is orthonormal. Throughout this paper, the $n \times n$ identify matrix is denoted by $I_{n}$, or simply $I$. The notation $A: i \times j$ means that $A$ is a matrix of size $i \times j$. For $\mathbf{x} \in \mathbf{R}^{d}$ we write $\mathbf{x}=\left(x_{1}, \ldots, x_{d}\right)^{T}$. 
In [17], we studied the multivariate orthogonal polynomials from the point of view that the orthogonality is given in terms of $V_{n}$ rather than a particular basis for $V_{n}$. One important characteristic of the orthogonal polynomials is the three-terms recurrence relation, as seen in the following multivariable version of the Favard's Theorem [17].

Theorem 2.1. Let $B=\left\{P_{j}^{k}\right\}_{k=0}^{\infty}{ }_{j=1}^{r_{k}}, P_{1}^{0} \neq 0$, be an arbitrary sequence in $\Pi^{d}$. Then the following statements are equivalent:

(1) There exists a linear functional $\mathscr{L}$ which induces a quasi-inner product in $\Pi^{d}$ and makes $\left\{\mathbb{P}_{k}\right\}_{k=0}^{\infty}$ an orthogonal basis in $\Pi^{d}$.

(2) For $k \geq 1,1 \leq i \leq d$, there exist matrices $A_{k, i}: r_{k} \times r_{k+1}, B_{k, i}: r_{k} \times r_{k}$, and $C_{k, i}: r_{k} \times r_{k-1}$, such that

(a) $x_{i} \mathbb{P}_{k}=A_{k, i} \mathbb{P}_{k+1}+B_{k, i} \mathbb{P}_{k}+C_{k, i} \mathbb{P}_{k-1}, \quad 1 \leq i \leq d$.

(b) $\operatorname{rank} A_{k}=r_{k+1}$, rank $C_{k+1}=r_{k+1}$,

where $A_{k}: d r_{k} \times r_{k+1}$ and $C_{k}: r_{k} \times d r_{k-1}$ are defined by

$$
A_{k}=\left[A_{k, 1}^{T}\left|A_{k, 2}^{T}\right| \cdots \mid A_{k, d}^{T}\right]^{T} \quad \text { and } C_{k}=\left[C_{k, 1}\left|C_{k, 2}\right| \cdots \mid C_{k, d}\right] .
$$

A weaker result appears in [9]. In this paper we study the recurrence relation with $C_{n, i}=A_{n-1, i}^{T}$. From Theorem 2.1 we have

Theorem 2.2. Let $\left\{\mathbb{P}_{n}\right\}_{n=0}^{\infty}, \mathbb{P}_{0}=1$, be a sequence in $\Pi^{d}$. Then the following statements are equivalent:

(1) There exists a linear functional which induces an inner product in $\Pi^{d}$ and makes $\left\{\mathbb{P}_{n}\right\}_{n=0}^{\infty}$ an orthonormal basis in $\Pi^{d}$.

(2) For $k \geq 0,1 \leq i \leq d$, there exist matrices $A_{k, i}: r_{k} \times r_{k+1}$ and $B_{k, i}$ : $r_{k} \times r_{k}$, such that

(a) $x_{i} \mathbb{P}_{k}=A_{k, i} \mathbb{P}_{k+1}+B_{k, i} \mathbb{P}_{n}+A_{k-1, i}^{T} \mathbb{P}_{k-1}, 1 \leq i \leq d$,

(b) $\operatorname{rank} A_{k}=r_{k+1}$, where $A_{-1, i}$ is taken to be zero.

Proof. If $\left\{\mathbb{P}_{n}\right\}_{n=0}^{\infty}$ is orthonormal with respect to an inner product $\mathscr{L}$, then $\mathscr{L}\left(\mathbb{P}_{n} \mathbb{P}_{n}^{T}\right)=I$ and $\left\{\mathbb{P}_{n}\right\}$ satisfies (a) by (2) in Theorem 2.1. On the other hand, if (2) holds, then from Theorem 2.1 there exists a quasi-inner product $\mathscr{L}$ which makes $\left\{\mathbb{P}_{n}\right\}_{n=0}^{\infty}$ orthogonal. We only need to show that the matrix $H_{n}=\mathscr{L}\left(\mathbb{P}_{n} \mathbb{P}_{n}^{T}\right)$ is the identity matrix for every $n \in \mathbf{N}_{0}$. Multiplying (a) by $\mathbb{P}_{k+1}$ and (a) with $k$ in place of $k-1$ by $\mathbb{P}_{k}$ respectively, and applying $\mathscr{L}$ leads to two formulas for $\mathscr{L}\left(x_{i} \mathbb{P}_{k} \mathbb{P}_{k+1}^{T}\right)$, which gives

$$
A_{k, i} H_{k+1}=H_{k} A_{k, i}, \quad 1 \leq i \leq d .
$$

Therefore,

$$
A_{k} H_{k+1}=G_{k} A_{k}, \quad G_{k}=\operatorname{diag}\left(H_{k}, \ldots, H_{k}\right) .
$$

Since $\mathbb{P}_{0}=1$, we have $H_{0}=$ [1]. Suppose $H_{k}$ is an identity matrix. Then $G_{k}$ is also an identity matrix. Thus, it follows from (b) and (2.3) that $H_{k+1}$ is an identity matrix. The proof is completed by induction.

We note that if $\mathscr{L}$ defines an inner product and $\left\{\mathbb{P}_{n}\right\}_{n=0}^{\infty}$ is an orthogonal basis in $\Pi^{d}$, then $H_{n}=\mathscr{L}\left(\mathbb{P}_{n} \mathbb{P}_{n}^{T}\right)$ is a positive definite matrix. Hence, there exists a nonsingular matrix $S_{n}$ such that $H_{n}=S_{n} S_{n}^{T}$. It is easy to verify that $\mathbf{Q}_{n}=S_{n}^{-1} \mathbb{P}_{n}$ satisfies the recurrence relation (a) in Theorem 2.2, and $\mathbf{Q}_{n}$ 
is an orthonormal basis for $V_{n}$. Therefore, from the point of view that the orthogonality is in terms of $V_{n}$, it is enough to study the simpler recurrence relation (a) in Theorem 2.2. In particular, it is enough for the purpose of analyzing the measure of orthogonality.

We note that $\left\{\mathbb{P}_{n}\right\}$ satisfying (a) in Theorem 2.2 is uniquely determined by the assumption $\mathbb{P}_{0}=1$ and the rank condition (b). The coefficient matrices in the recurrence relation satisfy the following matrix equations.

Corollary 2.3. In the statement (2) of Theorem 2.2, the matrices $A_{k, i}$ and $B_{k, i}$ satisfy the equations

$$
\begin{gathered}
A_{k, i} A_{k+1, j}=A_{k, j} A_{k+1, i}, \\
A_{k, i} B_{k+1, j}+B_{k, i} A_{k, j}=B_{k, j} A_{k, i}+A_{k, j} B_{k+1, i}, \\
A_{k-1, i}^{T}, A_{k-1, j}+B_{k, i} B_{k, j}+A_{k, i} A_{k, j}^{T} \\
=A_{k-1, j}^{T} A_{k-1, i}+B_{k, i} B_{k, j}+A_{k, j} A_{k, i}^{T},
\end{gathered}
$$

for $i \neq j, 1 \leq i, j \leq d$, and $k \geq 0$, where $A_{-1, i}=0$. Moreover, $B_{k, i}$ are symmetric.

Proof. By Theorem 2.2, there is an inner product $\mathscr{L}$ that makes $\mathbb{P}_{k}$ orthonormal. From the recurrence relation, we have two different ways of calculating the matrices $\mathscr{L}\left(x_{i} x_{j} \mathbb{P}_{k} \mathbb{P}_{k+2}^{T}\right), \mathscr{L}\left(x_{i} x_{j} \mathbb{P}_{k} \mathbb{P}_{k}^{T}\right)$, and $\mathscr{L}\left(x_{i} x_{j} \mathbb{P}_{k} \mathbb{P}_{k+1}^{T}\right)$. These calculations lead to the desired matrices equations. For examples, by the recurrence relation,

$$
\begin{aligned}
\mathscr{L}\left(x_{i} x_{j} \mathbb{P}_{k} \mathbb{P}_{k+2}^{T}\right) & =\mathscr{L}\left(x_{i} \mathbb{P}_{k} x_{j} \mathbb{P}_{k+2}^{T}\right) \\
& =\mathscr{L}\left[\left(A_{k, i} \mathbb{P}_{k+1}+\cdots\right)\left(\cdots+A_{k+1, j}^{T} \mathbb{P}_{k+1}\right)^{T}\right]=A_{k, i} A_{k+1, j},
\end{aligned}
$$

and

which leads to equation (2.4).

$$
\mathscr{L}\left(x_{i} x_{j} \mathbb{P}_{k} \mathbb{P}_{k+2}^{T}\right)=\mathscr{L}\left(x_{j} \mathbb{P}_{k} x_{i} \mathbb{P}_{k+2}^{T}\right)=A_{k, j} A_{k+1, i},
$$

The condition rank $A_{k}=r_{k+1}$ implies that there exist matrices $D_{k, i}: r_{k+1} \times$ $r_{k}, 1 \leq i \leq d, k \geq 0$, such that

$$
\sum_{i=1}^{d} D_{k, i} A_{k, i}=I
$$

\section{Main Results}

Let $\mathscr{M}=\mathscr{M}\left(\mathbf{R}^{d}\right)$ denote the set of nonnegative Borel measures $\mu$ on $\mathbf{R}^{d}$, defined on the $\sigma$-algebra of Borel sets, such that

$$
\int_{\mathbf{R}^{d}}\left|\mathbf{x}^{\alpha}\right| d \mu(\mathbf{x})<+\infty, \quad \forall \alpha \in \mathbf{N}_{0}^{d} .
$$

We are especially interested in the linear functional that has an integral representation

$$
\mathscr{L}(f)=\int_{\mathbf{R}^{d}} f(\mathbf{x}) d \mu(\mathbf{x}), \quad \mu \in \mathscr{M} .
$$


Such a $\mathscr{L}$ induces an inner product $\langle\cdot, \cdot\rangle$ by $\langle P, Q\rangle=\mathscr{L}(P Q)$. The measure $\mu$ is called a representation of $\mathscr{L}$. If $\left\{\mathbb{P}_{n}\right\}_{n=0}^{\infty}$ is orthogonal with respect to $\mathscr{L}$ expressible as in (3.1) we say that $\left\{\mathbb{P}_{n}\right\}_{n=0}^{\infty}$ is orthogonal with respect to the measure $\mu$. By the moments of $\mu \in \mathscr{M}$ we mean the numbers $\mu_{\alpha}=\int \mathbf{x}^{\alpha} d \mu(\mathbf{x})$, $\forall \alpha \in \mathbf{N}_{0}^{d}$. Two measures are called equivalent if they have the same moments. If the equivalent class of measures having the same moments as $\mu$ consists of $\mu$ only, the measure $\mu$ is called determinate, a terminology from the theory of moments, see $[3,7]$.

In contrast to one variable theory, Theorem 2.2 does not say whether the inner-product has a measure of representation. Our main result shows that such a measure exists and is unique, when the coefficient matrices satisfy a boundedness condition.

Let $\|\cdot\|_{2}$ be the spectral norm for matrices. It is induced by the Euclidean norm for vectors.

$$
\|A\|_{2}=\max \left\{\sqrt{\lambda}: \lambda \text { is an eigenvalue of } A^{T} A\right\} .
$$

Our main result is as follows.

Theorem 3.1. Let $\left\{\mathbb{P}_{n}\right\}_{n=0}^{\infty}, \mathbb{P}_{0}=1$, be a sequence in $\Pi^{d}$. Then the following statements are equivalent:

(i) There exists a determinate measure $\mu \in \mathscr{M}$ with compact support in $\mathbf{R}^{d}$ such that $\left\{\mathbb{P}_{n}\right\}_{n=0}^{\infty}$ is orthonormal with respect to $\mu$.

(ii) The statement (2) in Theorem 2.2 together with

$$
\sup _{k \geq 2}\left\|A_{k, i}\right\|_{2}<+\infty, \quad \sup _{k \geq 0}\left\|B_{k, i}\right\|_{2}<+\infty, \quad 1 \leq i \leq d .
$$

The proof of this theorem is in $\S 4$. It is based on the spectral theorem of a family of commuting selfadjoint operators. The connection between such a family of operators and multivariate orthogonal polynomials is the main establishment of the paper, and is of interest in itself. The operator approach is also used in the study of the moment problem (cf. $[3,7])$. Our results in $\S 4$ indicate that there is a close connection between the multivariable moment problem and orthogonal polynomials. We intend to study this connection in our future investigation. Let us also mention that the family of commuting selfadjoint operators plays an important role in the quantum mechanics (cf. [12]). It is interesting to see the possible applications of multivariate orthogonal polynomials in that direction.

For $d=1$; Theorem 3.1 is well known (cf. [5, p. 75 and p. 109]). In [9], an integral representation for the inner product is given, but the measure is not shown to be nonnegative nor determinate.

\section{THE OPERATOR APPROACH}

4.1. Spectral theory for commuting selfadjoint operators. We recall the part of spectral theory that will be needed, see $[1,2,12,13,14]$. Let $\mathscr{H}$ be a separable Hilbert space. Each selfadjoint operator $T$ in $\mathscr{H}$ has a unique spectral representation $T=\int x d E(x)$ where $E$ is the spectral measure, which is a projection valued measure defined for Borel sets of $\mathbf{R}$ such that $E(\mathbf{R})$ is the identity operator in $\mathscr{H}$ and $E(B \cap C)=E(B) \cap E(C)$ for Borel sets $B, C \subseteq \mathbf{R}$. For any $f \in \mathscr{H}$ the mapping $B \rightarrow\langle E(B), f, f\rangle$ is an ordinary measure defined for the Borel sets $B \subseteq \mathbf{R}$ and denoted $\langle E f, f\rangle$. 
The selfadjoint operators $T_{1}, \ldots, T_{d}$ in $\mathscr{H}$ with spectral measure $E_{1}, \ldots$, $E_{d}$, respectively, are said to be mutually commuting (mutually permutable in [13]) if their spectral measures commute, i.e.,

$$
E_{i}(B) E_{j}(C)=E_{i}(C) E_{j}(B)
$$

for any $i, j=1, \ldots, d$ and any two Borel sets $B, C \subseteq \mathbf{R}$. If $T_{1}, \ldots, T_{d}$ commute, then

$$
E=E_{1} \otimes \cdots \otimes E_{d}
$$

is a spectral measure on $\mathbf{R}^{d}$ with values that are selfadjoint projections in $\mathscr{H}$. In particular, $E$ is the unique measure such that

$$
E\left(B_{1} \times \cdots \times B_{d}\right)=E_{1}\left(B_{1}\right) \cdots E_{d}\left(B_{d}\right)
$$

for any Borel sets $B_{1}, \ldots, B_{d} \subseteq \mathbf{R}$. The measure $E$ is called the spectral measure of the commuting family $T_{1}, \ldots, T_{d}$.

We shall consider only bounded operators, see Remark 4.2 below. A vector $\Phi_{0} \in \mathscr{H}$ is a cyclic vector in $\mathscr{H}$ with respect to the commuting family of bounded selfadjoint operators $T_{1}, \ldots, T_{d}$ in $\mathscr{H}$ if the linear manifold $\left\{P\left(T_{1}, \ldots, T_{d}\right) \Phi_{0}, P \in \Pi^{d}\right\}$ is dense in $\mathscr{H}$. We summarize the spectral theorem for $T_{1}, \ldots, T_{d}$ in the following.

Theorem 4.1. Let $\mathscr{L}$ be a separable Hilbert space and $T_{1}, \ldots, T_{d}$ be a commuting family of bounded selfadjoint operators in $\mathscr{H}$. Let $S_{i}$ denote the spectrum of $T_{i}, 1 \leq i \leq D$. If $\Phi_{0}$ is a cyclic vector in $\mathscr{H}$ with respect to $T_{1}, \ldots, T_{d}$, then $T_{1}, \ldots, T_{d}$ are unitarily equivalent to the multiplication operators $X_{1}, \ldots, X_{d}$,

$$
\left(X_{i} f\right)(\mathbf{x})=x_{i} f(\mathbf{x}), \quad 1 \leq i \leq d,
$$

defined on $L^{2}\left(\mathbf{R}^{d}, \mu\right)$, where the measure $\mu$ is defined by $\mu(B)=\left\langle E(B) \Phi_{0}, \Phi_{0}\right\rangle$ for the Borel set $B \subset \mathbf{R}^{d}$ with support $S \subset S_{1} \times \cdots \times S_{d}$.

The unitary equivalence means that there exists a unitary mapping $U: \mathscr{H} \rightarrow$ $L^{2}\left(\mathbf{R}^{d}, \mu\right)$ such that

$$
U T_{i} U^{-1}=X_{i}, \quad 1 \leq i \leq d .
$$

We note that the measure $\mu$ satisfies the relation

$$
\left\langle\Phi_{0}, P\left(T_{1}, \ldots, T_{d}\right) \Phi_{0}\right\rangle=\int_{S} P(\mathbf{x}) d \mu(\mathbf{x})
$$

for all polynomials $P \in \Pi^{d}$. Since $T_{1}, \ldots, T_{d}$ are bounded, $S_{1}, \ldots, S_{d}$ are compact. Therefore $S$ is compact and $\mu$ is of compact support.

Remark: For bounded selfadjoint operators $T_{1}$ and $T_{2},(4.1)$ is a sufficient and necessary condition for $T_{1} T_{2}=T_{2} T_{1}$. However, for unbounded operators it has been shown by Nelson [11] that there are selfadjoint operators $T_{1}$ and $T_{2}$ with a common dense domain such that $T_{1} T_{2}=T_{2} T_{1}$, but their spectral measures do not commute.

4.2. The definition of the operators. Let $\mathscr{H}$ be a separable Hilbert space with fixed orthonormal basis $\left\{\psi_{n}\right\}_{n=0}^{\infty}$. For our purpose we shall rewrite $\left\{\psi_{n}\right\}_{n=0}^{\infty}$ as $\left\{\psi_{n}\right\}_{n=0}^{\infty}=\left\{\phi_{j}^{k}\right\}_{j=1}^{r_{k}} \underset{k=0}{\infty}$, where $r_{k}=r_{k}^{d}=\left(\begin{array}{c}k+d-1 \\ k\end{array}\right)$ for a fixed positive integer $d$ as before. We introduce the formal vector notation

$$
\Phi_{k}=\left[\phi_{1}^{k}, \ldots, \phi_{r_{k}}^{k}\right]^{T}, \quad k \in \mathbf{N}_{0} .
$$


For our convenience we shall say that $\left\{\Phi_{n}\right\}_{n=0}^{\infty}$ is orthonormal, formally

$$
\left\langle\Phi_{k}, \Phi_{m}^{T}\right\rangle=\left(\left\langle\phi_{i}^{k}, \phi_{j}^{m}\right\rangle\right)_{i=1}^{r_{k}}{ }_{j=1}^{r_{m}}=\delta_{i j} \delta_{k m} I_{r_{k}} .
$$

For every $f \in \mathscr{H}$ we can write in the vector-matrix notation that

$$
f=\sum_{k=0}^{\infty} \mathbf{a}_{k}^{T} \Phi_{k}, \quad \mathbf{a}_{k} \in \mathbf{R}^{r_{k}} .
$$

If $T: \mathscr{H} \rightarrow \mathscr{H}$ is a linear operator, we mean by $T \Phi_{k}$ the vector

$$
T \Phi_{k}=\left[T \phi_{1}^{k}, \ldots, T \phi_{r_{k}}^{k}\right]^{T} .
$$

We denote by $F_{k, m}$ the matrix

$$
F_{k, m}=\left(\left\langle T \Phi_{k}, \Phi_{m}^{T}\right\rangle\right)=\left(\left\langle T \phi_{i}^{k}, \phi_{j}^{m}\right\rangle\right)_{i=1}^{r_{k}} \substack{m \\ j=1}_{j} .
$$

Then we can write

$$
T f=\sum_{k=0}^{\infty} \mathbf{b}_{k}^{T} \Phi_{k}, \quad \mathbf{b}_{k}^{T}=\sum_{j=0}^{\infty} \mathbf{a}_{j}^{T} F_{j, k} .
$$

The usual matrix representation of the linear operator now takes the form $T=$ $\left(F_{i j}\right)_{i, j=1}^{\infty}$. The elements of $T$ are matrices $F_{i j}: r_{i} \times r_{j}$, whose sizes are different for different pairs of $(i, j)$. Note that we have used the same symbol for both the operator and its matrix representation.

We now define the linear operators associated with the recurrence relation in Theorem 2.2. Let $A_{k, i}: r_{k} \times r_{k+1}$ and $B_{k, i}: r_{k} \times r_{k}$ be given matrices such that the rank condition (b) in Theorem 2.2 is satisfied. Furthermore, assume that $B_{k, i}$ are symmetric, and $A_{k, i}$ and $B_{k, i}$ satisfy equations (2.4), (2.5), and (2.6). We then define $T_{i}: \mathscr{H} \rightarrow \mathscr{H}, 1 \leq i \leq d$, to be the linear operator whose matrix representation with respect to $\left\{\Phi_{k}\right\}_{k=0}^{\infty}$ is given by

$$
T_{i}=\left[\begin{array}{cccc}
B_{0, i} & A_{0, i} & & \bigcirc \\
A_{0, i}^{T} & B_{1, i} & A_{1, i} & \\
& A_{1, i}^{T} & B_{2, i} & \ddots \\
\bigcirc & & \ddots & \ddots
\end{array}\right], \quad 1 \leq i \leq d .
$$

We can consider $T_{i}$ as matrix operator which acts on sequences in $l^{2}$ via matrix multiplication. For $d=1$, we have $r_{k}=1$ for all $k \in \mathbf{N}_{0}$ and the matrix $T_{1}$ is the Jacobi matrix (cf. [15]).

4.3. Properties of $T_{i}$. We restrict ourselves to the bounded operators.

Lemma 4.3. The operator $T_{i}$ is bounded if and only if $\sup _{k \geq 0}\left\|A_{k, i}\right\|_{2}<\infty$ and $\sup _{k \geq 0}\left\|B_{k, i}\right\|_{2}<\infty$.

Proof. For any $f \in \mathscr{H}, f=\sum \mathbf{a}_{k}^{T} \Phi_{k}$, we have $\|f\|_{\mathscr{L}}^{2}=\langle f, f\rangle=\sum \mathbf{a}_{k}^{T} \mathbf{a}_{k}$. It follows easily from the definition that

$$
\begin{aligned}
T_{i} f & =\sum_{k=0}^{\infty} \mathbf{a}_{k}^{T}\left[A_{k, i} \boldsymbol{\Phi}_{k+1}+B_{k, i} \Phi_{k}+A_{k-1, i}^{T} \Phi_{k-1}\right] \\
& =\sum_{k=0}^{\infty}\left[\mathbf{a}_{k-1}^{T} A_{k-1, i}+\mathbf{a}_{k}^{T} B_{k, i}+\mathbf{a}_{k+1}^{T} A_{k, i}^{T}\right] \Phi_{k}
\end{aligned}
$$


where we define $A_{-1, i}=0$. Therefore, if $\sup _{k \geq 0}\left\|A_{k, i}\right\|_{2}<+\infty$ and $\sup _{k \geq 0}\left\|B_{k, i}\right\|_{2}<+\infty$, then

$$
\begin{aligned}
\left\|T_{i} f\right\|_{\mathscr{P}}^{2} & =\sum_{k=0}^{\infty}\left\|\mathbf{a}_{k-1}^{T} A_{k-1, i}+\mathbf{a}_{k}^{T} B_{k, i}+\mathbf{a}_{k+1}^{T} A_{k, i}^{T}\right\|_{2}^{2} \\
& \leq 3\left(2 \sup _{k \geq 0}\left\|A_{k, i}\right\|_{2}^{2}+\sup _{k \geq 0}\left\|B_{k, i}\right\|_{2}^{2}\right)\|f\|_{\mathscr{P}}^{2} .
\end{aligned}
$$

Thus, $T_{i}$ is a bounded operator. Conversely, suppose that $\left\|A_{k, i}\right\|_{2}$, say, goes to infinity for a subsequence of $\mathbf{N}_{0}$. Let $\mathbf{a}_{k}$ be vectors such that $\left\|\mathbf{a}_{k}\right\|_{2}=1$ and $\left\|\mathbf{a}_{k}^{T} A_{k, i}\right\|_{2}=\left\|A_{k, i}\right\|_{2}$. Then we have $\left\|\mathbf{a}_{k} \Phi_{k}\right\|=\left\|\mathbf{a}_{k}\right\|_{2}=1$. Therefore, it follows from

$$
\left\|T_{i}\right\|_{\mathscr{P}}^{2} \geq\left\|T_{i} \mathbf{a}_{k}^{T} \Phi_{k}\right\|_{\mathscr{P}}^{2}=\left\|\mathbf{a}_{k}^{T} A_{k, i}\right\|_{2}^{2}+\left\|\mathbf{a}_{k}^{T} B_{k, i}\right\|_{2}^{2}+\left\|\mathbf{a}_{k}^{T} A_{k-1, i}^{T}\right\|_{2}^{2} \geq\left\|A_{k, i}\right\|_{2}^{2}
$$

that $T_{i}$ is unbounded.

Lemma 4.4. Suppose $T_{i}, 1 \leq i \leq d$, are bounded. Then $T_{i}, 1 \leq i \leq d$, are selfadjoint operators, and $T_{1}, \ldots, T_{d}$ commute.

Proof. Since $T_{i}$ is bounded, it is selfadjoint if it is symmetric, i.e., $\left\langle T_{i} f, g\right\rangle=$ $\left\langle f, T_{i} g\right\rangle$. But this follows from the obvious symmetry of the matrix representation (4.7). To prove that $T_{1}, \ldots, T_{d}$ commute, we only need to show

$$
T_{k} T_{j} f=T_{j} T_{k} f, \quad \forall f \in \mathscr{H},
$$

since $T_{i}$ 's are bounded. A simple calculation shows that this is equivalent to the matrix equations (2.4), (2.5), and (2.6), which are assumed.

Lemma 4.5. Suppose $T_{1}, \ldots, T_{d}$ are bounded operators. Then $\Phi_{0} \in \mathscr{H}$ is a cyclic vector with respect to $T_{1}, \ldots, T_{d}$, and

$$
\Phi_{n}=\mathbb{P}_{n}\left(T_{1}, \ldots, T_{d}\right) \Phi_{0}
$$

where $\mathbb{P}_{n}\left(x_{1}, \ldots, x_{d}\right)$ is of the form (2.2).

Proof. If (4.8) is true, then $\Phi_{0}$ is a cyclic vector by definition. To prove (4.8), we use induction. Clearly $\mathbb{P}_{0}=1$. From the definition of $T_{i}$ we have

$$
T_{i} \Phi_{0}=A_{0, i} \Phi_{1}+B_{0, i} \Phi_{0}, \quad 1 \leq i \leq d .
$$

Therefore,

$$
A_{0, i} \Phi_{1}=T_{i} \Phi_{0}-B_{0, i} \Phi_{0}, \quad 1 \leq i \leq d .
$$

Multiply this equation by $D_{0, i}$ in (2.7) and sum for $i=1, \ldots, d$, we get

$$
\Phi_{1}=\sum_{i=1}^{d} D_{0, i} T_{i} \Phi_{0}-\sum_{i=1}^{d} D_{0, i} B_{0, i} \Phi_{0}=\left(\sum_{i=1}^{d} T_{i} D_{0, i}-E_{0}\right) \Phi_{0}
$$

where $E_{0}=\sum D_{0, i} B_{0, i}$. Therefore

$$
\mathbb{P}_{1}(\mathbf{x})=\sum_{i=1}^{d} \lambda_{i} D_{0, i}-E_{0}
$$

Since $D_{0, i}$ is of the size $r_{1} \times r_{0}=d \times 1, \mathbb{P}_{1}$ is of the desired form. Likewise, for $k \geq 1$,

$$
T_{i} \boldsymbol{\Phi}_{k}=A_{k, i} \boldsymbol{\Phi}_{k+1}+B_{k, i} \boldsymbol{\Phi}_{k}+A_{k-1, i}^{T} \boldsymbol{\Phi}_{k-1}, \quad 1 \leq i \leq d,
$$


therefore

$$
\Phi_{k+1}=\sum_{i=1}^{d} T_{i} D_{k, i} \Phi_{k}-E_{k} \Phi_{k}-F_{k} \Phi_{k-1}
$$

where

$$
E_{k}=\sum_{i=1}^{d} D_{k, i} B_{k, i} \quad \text { and } \quad F_{k}=\sum_{i=1}^{d} D_{k, i} A_{k-1, i}^{T} .
$$

By induction we then have

$$
\Phi_{k+1}=\left[\sum_{k=1}^{d} T_{i} D_{k, i} \mathbb{P}_{k}(T)-E_{k} \mathbb{P}_{k}(T)-F_{k} \mathbb{P}_{k-1}(T)\right] \Phi_{0},
$$

where $T=\left(T_{1}, \ldots, T_{d}\right)^{T}$. Therefore

$$
\mathbb{P}_{k+1}(\mathbf{x})=\sum_{k=1}^{d} \lambda_{i} D_{k, i} \mathbb{P}_{k}(\mathbf{x})-E_{k} \mathbb{P}_{k}(\mathbf{x})-F_{k} \mathbb{P}_{k-1}(\mathbf{x}) .
$$

Clearly, every component of $\mathbb{P}_{k+1}$ is a polynomial in $\Pi_{k+1}$.

From these lemmas and Theorem 4.1, we have proved

Theorem 4.6. If $\sup _{k \geq 0}\left\|A_{k, i}\right\|_{2}<\infty$ and $\sup _{k \geq 0}\left\|B_{k, i}\right\|_{2}<\infty$ for $1 \leq i \leq d$, then there exists a measure $\mu \in \mathscr{M}$ with compact support such that $T_{1}, \ldots, T_{d}$ are unitarily equivalent to the multiplication operators $X_{1}, \ldots, X_{d}$ in $L^{2}\left(\mathbf{R}^{d}, \mu\right)$.

4.4. Proof of the main theorem. The unitary equivalence in Theorem 4.6 associates the cyclic vector $\Phi_{0}$ with the function $f(\mathbf{x})=1$ and $\left(T_{1}^{\alpha_{1}} \cdots T_{d}^{\alpha_{d}}\right) \Phi_{0}$ with $f(\mathbf{x})=\mathbf{x}^{\alpha}$. Moreover, the orthonormal basis $\left\{\Phi_{n}\right\}_{n=0}^{\infty}$ in $\mathscr{H}$ corresponds to $\left\{\mathbb{P}_{n}\right\}_{n=0}^{\infty}$ in $L^{2}\left(\mathbf{R}^{d}, \mu\right)$ as shown in (4.8). We have

Lemma 4.7. The polynomials $\left\{\mathbb{P}_{n}\right\}_{n=0}^{\infty}$ in Lemma 4.5 are orthonormal with respect to $\mu$, and they satisfy the recurrence relation (a) in Theorem 2.2 with the matrices in (4.7) as the coefficients.

Proof. Since $\mu(B)=\left\langle E(B) \Phi_{0}, \Phi_{0}\right\rangle$ in Theorem 4.1, we have by (4.4) that

$$
\int \mathbb{P}_{n}(\mathbf{x}) \mathbb{P}_{m}^{T}(\mathbf{x}) d \mu(\mathbf{x})=\left\langle\mathbf{P}_{n} \Phi_{0}, \mathbb{P}_{m}^{T} \Phi_{0}\right\rangle=\left\langle\Phi_{n}, \Phi_{m}^{T}\right\rangle
$$

This proves that $\left\{\mathbb{P}_{n}\right\}$ are orthonormal. From Theorem 2.2 the polynomials $\left\{\mathbb{P}_{n}\right\}$ satisfy a recurrence relation of the form (a) in that theorem. By the unitary equivalence in Theorem 4.6, the multiplication operators $X_{1}, \ldots, X_{d}$ at (4.2) have the same matrix representation (4.7) with respect to $\left\{\mathbb{P}_{n}\right\}$ in $L^{2}\left(\mathbf{R}^{d}, \mu\right)$. Since $X_{i} \mathbb{P}_{n}=x_{i} \mathbb{P}_{n}$, the coefficient matrices in the recurrence relation that $\left\{\mathbb{P}_{n}\right\}$ satisfy are the same matrices in (4.7).

Proof of Theorem 3.1. (i) $\Rightarrow$ (ii). From Theorem 2.2, we only need to prove (3.2). However, since $\mu$ has compact support, the multiplication operators $X_{1}, \ldots, X_{d}$ in $L^{2}\left(\mathbf{R}^{d}, \mu\right)$ are bounded. Since $X_{1}, \ldots, X_{d}$ have the matrix representation (4.7) with respect to $\left\{\mathbb{P}_{n}\right\},(3.2)$ follows from Lemma 4.3.

(ii) $\Rightarrow$ (i). If $\left\{\mathbb{P}_{n}\right\}$ satisfies the recurrence relation in the theorem, then we can use the coefficient matrices to define operators $T_{i}$ through (4.7). By Lemma 4.7, the polynomials in Lemma 4.5 satisfy the same recurrence relation. Since 
the recurrence relation determinates uniquely the polynomials under the consideration, the $\left\{\mathbb{P}_{n}\right\}$ are the polynomials in Lemma 4.5. Therefore, the existence of the measure $\mu$ with compact support has been established by our previous results. That $\mu$ is determinate follows from the boundedness of the multiplication operators and [3, Theorem 4].

\section{ACKNOWLEDGMENT}

The author thanks one referee for clarifying one inaccurate point in the manuscript.

\section{REFERENCES}

1. N. I. Akhiezer and I. M. Glazman, Theory of linear operators in Hilbert space, Ungar, New York, 1961.

2. S. K. Berberian, Notes on spectral theory, Van Nostrand, Princeton, N.J., 1966.

3. C. Berg, The multidimensional moment problem and semigroups, Moments in Mathematics, Proc. Sympos. Appl. Math., Vol. 37, Amer. Math. Soc., Providence, R.I., 1987, pp. 110-124.

4. M. Bertran, Notes on orthogonal polynomials in $\nu$-variables, SIAM J. Math. Anal. 6 (1975), 250-257.

5. T. S. Chihara, An introduction to orthogonal polynomials, Math. and its Appl., Vol. 13, Gordon and Breach, New York, 1978.

6. J. Dombrowski, Orthogonal polynomials and functional analysis, Orthogonal Polynomials (P. Nevai, ed.), Kluwer Academic, Amsterdam, 1990, pp. 147-161.

7. $ـ$ Tridiagonal matrix representations of cyclic selfadjoint operators, I, Pacific J. Math. 114 (1984), 325-334; II 120 (1985), 47-53.

8. B. Fuglede, The multidimensional moment problem, Math. Expositions 1 (1983), 47-65.

9. M. A. Kowalski, Orthogonality and recursion formulas for polynomials in $n$ variables, SIAM J. Math. Anal. 13 (1982), 316-323.

10. H. L. Krall and I. M. Sheffer, Orthogonal polynomials in two variables, Ann. Mat. Pura Appl. (4) 76 (1967), 325-376.

11. E. Nelson, Analytic vectors, Ann. of Math. (2) 70 (1959), 572-615.

12. E. Prugovecki, Quantum mechanics in Hilbert space, 2nd ed., New York, 1981.

13. F. Riesz and B. Sz.-Nagy, Functional analysis, Ungar, New York, 1955.

14. B. D. Sleeman, Multiparameter spectral theory in Hilbert Spaces, Pitman, London, 1978.

15. M. Stone, Linear transformations in Hilbert space and their applications to analysis, Amer. Math. Soc. Colloq. Publ., Vol. 15, Amer. Math. Soc., Providence, R.I., 1932; reprinted 1983.

16. G. Szegö, Orthogonal polynomials, Amer. Math. Soc. Colloq. Publ., Vol. 23, Amer. Math. Soc., Providence, R.I., 4th ed., 1975.

17. Y. Xu, On multivariate orthogonal polynomials, SIAM J. Math. Anal. 24 (1993), 783-794.

Department of Mathematics and Statistics, University of Arkansas at Little Rock, LitTle Rock, Arkansas 72204

Current address: Department of Mathematics, University of Oregon, Eugene, Oregon 97403

E-mail address: yuan@bright.uoregon.edu 